\title{
Aminocyclopyrachlor e indaziflam: Seletividade, controle e comportamento no ambiente $^{1}$
}

\author{
Aminocyclopyrachlor and indaziflam: Selectivity, control and fate in the \\ environment
}

\author{
Naiara Guerra ${ }^{2}$; Rubem Silvério de Oliveira Júnior ${ }^{3}$; Jamil Constantin ${ }^{3}$; Antonio Mendes de \\ Oliveira Neto ${ }^{2}$; Guilherme Braga Pereira Braz ${ }^{4}$
}

\begin{abstract}
Resumo - Os herbicidas aminocyclopyrachlor e indaziflam são duas novas moléculas que estão em fase de desenvolvimento no Brasil. Aminocyclopyrachlor é um mimetizador de auxina, e o indaziflam atua na biossíntese de parede celular e ambos apresentam grande potencial para controle de plantas daninhas em pré-emergência. O aminocyclopyrachlor é seletivo para espécies pertencentes à família Poaceae, enquanto que o indaziflam é pouco seletivo para culturas anuais, estando em desenvolvimento para culturas perenes. Embora apresentem características distintas de sorção ao solo, ambos herbicidas apresentam correlação positiva entre a sorção e o conteúdo de matéria orgânica. A longa persistência da atividade residual no solo das duas moléculas exige que ambas sejam utilizadas com cautela, em função do potencial de carryover.
\end{abstract}

Palavras-chaves: ácidos pirimidinecarboxílicos, alkylazine, inibidores da síntese de parede celular, mimetizadores de auxina

Abstract - Aminocyclopyrachlor and indaziflam are two new molecules that are currently under development in Brazil. Aminocyclopyrachlor is an auxinic herbicide, and indaziflam acts in the biosynthesis of cell wall and both herbicides are expected to have great potential for preemergence weed control. Aminocyclopyrachlor is selective to the species belonging to the Poaceae family, while indaziflam is less selective for annual crops, and is under development for perennial crops. Although the two herbicides pose distinct properties related to sorption to soil, both present positive correlation between sorption and organic matter contents. The long persistence of residual activity in soil of these two molecules requires that they be used with caution, because of their carryover potential.

Keywords: Pyrimidinecarboxylic acid, alkylazine, inhibiting cellulose biosynthesis inhibitor, auxin herbicides

\footnotetext{
${ }^{1}$ Recebido para publicação em 03/03/2014 e aceito em 15/04/2014.

2 Docente do curso de Agronomia da Faculdade Integrado de Campo Mourão - PR. Endereço para correspondência: Rodovia BR 158, KM 207, s/n, CEP 87300-970, Campo Mourão, Paraná, Brasil. Email: <naiaraguerra.ng@gmail.com>.

${ }^{3}$ Docente do curso de Agronomia da Universidade Estadual de Maringá - UEM.

${ }^{4}$ Doutorando do Programa de Pós-graduação em Agronomia da Universidade Estadual de Maringá - PGA/UEM.
} 


\section{Introdução}

O desenvolvimento de uma nova molécula herbicida é um processo complexo e que demanda muito investimento. Por este motivo, nos últimos anos foram poucas as novas moléculas desenvolvidas e registradas para uso comercial. Este processo ainda é mais lento e oneroso quando se trata de produtos que apresentem grupos químicos ou mecanismos de ação ainda não presentes no país.

As novas moléculas herbicidas aminocyclopyrachlor e indaziflam encontramse atualmente em fase de desenvolvimento no Brasil. O primeiro possui registro nos Estados Unidos para o controle de plantas daninhas dicotiledôneas e arbustos em áreas não agrícolas e pastagens; o segundo foi registrado nos Estados Unidos e Canadá para uso em gramados e em áreas cultivadas com espécies frutíferas (Turner et al., 2009; Kaapro \& Hall, 2012; Bayer, 2012).

Aminocyclopyrachlor é um herbicida mimetizador de auxina, do novo grupo químico dos ácidos pirimidinecarboxílicos e o indaziflam pertence a também nova classe química denominada alkylazine, e atua na biossíntese de parede celular. Ambos apresentam atividade em pré e pós-emergência, mas quando aplicados ao solo apresentam prolongado efeito residual.

Em virtude da intensificação do número de casos de plantas daninhas resistentes no Brasil, estas duas novas moléculas eventualmente terão grande aplicabilidade no manejo de plantas daninhas, pois como são moléculas de grupos químicos ainda não registrados no Brasil. No caso do indaziflam, embora não apresente um mecanismo de ação inédito, nenhum outro herbicida com este mecanismo de ação encontra-se atualmente registrado para uso no Brasil.

A presente revisão teve como objetivo compilar as informações existentes sobre a seletividade, eficácia e comportamento no ambiente dos herbicidas aminocyclopyrachlor e indaziflam, uma vez que possivelmente em curto espaço de tempo estes estarão liberados para o uso em nosso país.

\section{Aminocyclopyrachlor}

O aminocyclopyrachlor (Ácido 6amino-5-cloro-2-ciclopropilpirimidino-4carboxílico) é um herbicida mimetizador de auxina, do novo grupo químico dos ácidos pirimidinecarboxílicos. Foi registrado nos Estados Unidos no ano de 2010, para o controle de plantas daninhas eudicotiledôneas e arbustos em áreas não agrícolas e pastagens, incluindo gramados, aeroportos, rodovias, ferrovias e áreas de recreação (Turner et al., 2009), e apresenta tanto atividade foliar quanto no solo (Rick et al., 2008; Turner et al., 2009; Mc Closkey et al., 2011). Várias espécies em numerosas famílias eudicotiledôneas são sensíveis ao aminocyclopyrachlor, incluindo Asteraceae, Fabaceae, Chenopodiaceae, Convolvulaceae e Euphorbiaceae (Claus et al., 2008; Armel et al., 2009; Turner et al., 2009; Jenks, 2010).

Aminocyclopyrachlor apresenta seletividade para muitas espécies Liliopsidas (Edward, 2008; Vassios et al., 2009; Guerra et al., 2014) e normalmente estas plantas possuem maior tolerância quando comparada com espécies Magnoliopsidas (Strachan et al., 2010; Guerra et al., 2014). Após avaliação da sensibilidade de oito espécies ao aminocyclopyrachlor, concluiu-se que sorgo, milho e milheto não sofreram qualquer tipo de injúrias quando semeadas em solos que receberam a aplicação de até $50 \mathrm{~g} \mathrm{ha}^{-1}$ deste herbicida. No entanto, as culturas do algodão, soja, girassol, pepino e beterraba apresentaram sintomas de intoxicação caracterizados por epinastia e enrolamento das folhas, ramos e pecíolo, além de apresentarem redução significativa na massa fresca da parte aérea (Guerra et al., 2014). Os sintomas deste herbicida em plantas sensíveis caracterizam-se por epinastia dos caules e folhas recémdesenvolvidas e perda da dominância apical (Grossnann, 2009; Strachan et al., 2010). 
Nenhuma espécie Liliopsida mostrou-se sensível ao aminocyclopyrachlor, de modo que nem mesmo a aplicação no solo de $64 \mathrm{~g} \mathrm{ha}^{-1}$ deste herbicida foi suficiente para atingir valores de $\mathrm{I}_{25}$ (redução de $25 \%$ no acúmulo de massa seca em relação à testemunha sem herbicida) dentre as espécies testadas por Strachan et al. (2010; 2011). Entre tais espécies encontravam-se milho, aveia, trigo, azevém e cevada. Estas espécies são tolerantes à maioria dos herbicidas mimetizadores de auxinas, e a seletividade está relacionada a diversos fatores, como arranjo do tecido vascular em feixes, presença de meristemas intercalares, metabolização e exsudação via sistema radicular. Desta forma, o movimento das auxinas nestas espécies é mais restrito, ocorrendo a degradação e metabolização de forma mais acentuada (Flessner et al., 2011) o que leva à menor chegada destes produtos nos locais onde atuam, ou seja, os meristemas em crescimento nas plantas.

A absorção e translocação do aminocyclopyrachlor dependem da sensibilidade da planta-alvo. Espécies altamente suscetíveis como Lactuca serriola absorvem e translocam menos herbicida do que espécies relativamente menos sensíveis, como a espécie perene Chondrilla juncea (Bell et al., 2011). A translocação ocorre através do floema, de forma semelhante a outros herbicidas auxínicos (Bukun et al., 2010), se acumulando nos tecidos meristemáticos (Bell et al., 2011).

Em Magnoliopsidas a absorção e translocação deste herbicida são significativas, resultando em controle eficiente de espécies de plantas daninhas importantes em regiões de clima temperado, tais como Cirsium arvense, Fallopia japonica, Hypochaeris radicata, Plantago spp., entre outras (Bukun et al., 2010). Estudos desenvolvidos por Lindenmayer et al. (2013) demonstraram que o aminocyclopyrachlor é rapidamente absorvido pelas folhas de Convolvulus arvensis, onde 12,6 horas após a aplicação, cerca de $90 \%$ do total do herbicida aplicado havia sido absorvido, sendo esta velocidade de absorção superior à de outros herbicidas com o mesmo mecanismo de ação, como 2,4-D, picloram (Agbakoba \& Goodin, 1970) e dicamba (Flint \& Barrett, 1989). Este herbicida também é uma alternativa eficiente para o controle de algumas espécies resistentes aos herbicidas glyphosate e inibidores da ALS, como Conyza canadensis, Kochia scoparia, Lactuca serriolada e Salsola spp. (Claus et al., 2009; Turner et al., 2010). Para Lolium arundinaceum, espécie da família Poaceae, foram necessárias 3 e 48 horas após a aplicação do aminocyclopyrachlor para se atingir porcentagens de absorção de 38 e 68\%, respectivamente. Para esta espécie a translocação ficou limitada à folha na qual o herbicida foi aplicado (Lewis et al., 2013).

As doses utilizadas em trabalhos de pesquisa têm variado de 35 a $315 \mathrm{~g} \mathrm{ha}^{-1}$. No entanto, para espécies sensíveis doses entre 70 e $140 \mathrm{~g} \mathrm{ha}^{-1}$ tem sido suficientes para proporcionar controle eficiente. Tais doses são bem menores do que as comumente utilizadas por outros herbicidas auxínicos, como o 2,4-D, possibilitando menor impacto ao ambiente e intoxicação de mamíferos, mesmo ainda assim sendo esperados longos períodos de atividade residual (Westra et al., 2008; Turner et al., 2009). Observações realizadas a campo indicam que aminocyclopyrachlor é um herbicida muito eficaz para o controle de Convolvulus arvensis, atingindo níveis superiores a $90 \%$ durante até 16 meses após a aplicação de $35 \mathrm{~g} \mathrm{ha}^{-1}$, superando os herbicidas quinclorac e picloram (Lindenmayer et al., 2009).

Um dos aspectos relevantes para compreender o potencial de utilização do aminocyclopyrachlor passa pela compreensão do seu comportamento no solo. Apesar do controle residual de diversas espécies de plantas daninhas, o aminocyclopyrachlor deve ser utilizado com cautela, pois pode prejudicar as espécies que serão cultivadas posteriormente (Kniss \& Lyon, 2011). Têm-se como exemplo 
a cultura do trigo, que não apresentou sintomas de lesões visíveis após a semeadura em solos que receberam $20 \mathrm{~g} \quad \mathrm{ha}^{-1} \mathrm{de}$ aminocyclopyrachlor 15 dias antes da semeadura, no entanto, no momento da colheita observou-se redução de $50 \%$ na produtividade de grãos. As culturas da alfafa, soja e girassol também apresentam sensibilidade ao carryover em solos que receberam a aplicação de aminocyclopyrachlor (Westra et al., 2008).

As principais características físicoquímicas do aminocyclopyrachlor são descritas na Tabela 1. Existe correlação positiva entre a sorção do aminocyclopyrachlor e os teores de matéria orgânica e argila do solo em solos brasileiros e americanos (Oliveira $\mathrm{Jr}$ et al., 2011). Embora seja considerado um herbicida ácido fraco (pKa 4,65), não se observou correlação significativa entre $K f$ e o pH do solo para o intervalo de $\mathrm{pH}$ entre 4,9 e 7,2. Neste trabalho, como os níveis de $\mathrm{pH}$ do solo estavam acima do $\mathrm{pKa}$ do aminocyclopyrachlor, este encontrava-se substancialmente dissociado e muito pouco sorvido. Desta forma, com base no pKa deste herbicida é esperada baixa sorção para a maioria dos solos estudados. Lindenmayer (2012) também demonstrou que a sorção do aminocyclopyrachlor tem relação positiva com os teores de matéria orgânica e silte, assim como não há correlação entre a sorção e o pH do solo. Em relação ao potencial teórico de lixiviação para o lençol freático, estudo baseado na estimativa do coeficiente GUS concluiu que o aminocyclopyrachlor apresentou mobilidade semelhante ao picloram, e que este herbicida poderia ser considerado como lixiviável se a meia-vida no solo excedesse 12,7 dias (Oliveira Jr. et al., 2012).

Tabela 1. Características físico-químicas das moléculas de aminocyclopyrachlor e indaziflam. Características Aminocyclopyrachlor Indaziflam

Estrutura química<smiles>Nc1nc(C2CC2)nc(C(=O)O)c1Cl</smiles>

Ácido 6-amino-5-cloro-2-

Nome químico ciclopropilpirimidino-4-

Solubilidade $\left(\mathrm{mg} \mathrm{L}^{-1}\right)$

$\mathrm{pKa}^{1}$

$\mathrm{K}_{\mathrm{ow}}{ }^{2}$

$\mathrm{K}_{\mathrm{oc}}{ }^{3}\left(\mathrm{mg} \mathrm{g}^{-1}\right)$

$\mathrm{K}_{\mathrm{f}}^{4}$

Meia vida $-\mathrm{t}_{1 / 2}$ (dias)

Pressão de Vapor (Pa)

Degradação

${ }^{1}$ constante de equilíbrio de ionização ácido; ${ }^{2}$ coeficiente de partição octanol-água; ${ }^{3}$ coeficiente de partição normalizado para o teor de carbono orgânico; ${ }^{4}$ coeficiente de sorção de Freundlich; Informações extraídas de trabalhos de Finkelstein et al. (2008); Lindenmayer et al. (2009); U.S. EPA (2010); Bayer (2010); Alonso et al. (2011) e Oliveira Jr. et al. (2011). carboxílico

N-[(1R,2S)-2,3-dihidro-2,6dimetil-1H-inden-1-il]-6-[(1R)1-fluoroetilo]-1,3,5-triazina2,4-diamina $4,4(\mathrm{pH} \mathrm{4,0)}$ $2,2(\mathrm{pH} 7,0-9,0)$ $4200(\mathrm{pH} \mathrm{4,0)}$
$2800(\mathrm{pH} \mathrm{7,0-9,0)}$ 4,65 2,48 3,50 2,80 $<1000$

$0,06-1,64$ $37-128$ $4,89 \times 10^{-6}$ $4,66-29,3$ $>150$ $6,92 \times 10^{-8}$ 
A degradação do aminocyclopyrachlor pode ser mais lenta em solos com baixo teor de matéria orgânica e maior teor de argila, devido a maior quantidade de microorganismos no solo com maior teor de matéria orgânica (Conklin \& Lym, 2013).

Segundo Finkelstein et al. (2008), o aminocyclopyrachlor apresenta meia-vida $\left(t_{1 / 2}\right)$ no solo de 72-128 dias. No entanto Lindenmayer (2012) verificou valores de meiavida de 32,5 dias, provavelmente devido a alta umidade proporcionada pelo sistema de irrigação ter favorecido a atividade microbiana, e em consequência, a degradação deste herbicida. Outros estudos indicam que o aminocyclopyrachlor sofre fotodegradação quando submetido a águas rasas expostas a luz solar, apresentando, nestas circunstâncias, meia-vida de 1,2 dias (Claus et al., 2008).

Lindenmayer (2012) mencionou que quando o aminocyclopyrachlor é aplicado no solo permanece em torno de 14 dias após a sua aplicação em grande concentração na camada superficial. Após 28 dias este tende a diminuir a sua concentração no solo e passar para camadas mais profundas, sendo possível a sua detecção 365 dias após a sua aplicação nas camadas de 5-15 e 15-30 cm de profundidade do solo. Com base nos coeficientes de sorção do aminocyclopyrachlor (Tabela 1), espera-se que este possa apresentar alta mobilidade no solo (Oliveira Jr et al., 2011).

Este herbicida foi desenvolvido inicialmente sob duas formulações, uma na forma ácida (aminocyclopyrachlor) e outra na forma metil éster (aminocyclopyrachlor éster metílico). Cerca de $80 \%$ do éster metílico é convertido para ácido livre em plantas seis horas após a absorção foliar (Bukun et al., 2010). Um dos problemas associados aos herbicidas auxínicos é a elevada pressão de vapor. Comparando as formulações de aminocyclopyrachlor e aminocyclopyrachlor éster metílico, verifica-se que o aminocyclopyrachlor apresenta baixa volatilidade, enquanto que a formulação éster metílico pode volatilizar-se a partir de superfícies não porosas como o vidro e o plástico. E apesar da volatilização do éster metílico poder ser reduzida com a utilização de tensoativos, as perdas na melhor das hipóteses foram superiores a $50 \%$ em condições de laboratório. Apesar de ambas as formulações serem igualmente ativas como herbicidas, a forma de éster de metílico tem uma pressão de vapor mais baixa e é mais volátil do que a forma ácida (Strachan et al., 2010). Resultados de Strachan et al. (2010; 2013) sugerem que o risco de volatilidade e movimento subsequente de vapor do aminocyclopyrachlor éster metílico é intermediário entre o do dicamba e aminopyralid, ao passo que o risco de volatilidade e movimento subsequente de vapor do aminocyclopyrachlor parece ser semelhante ou ainda menor do que o do aminopyralid.

\section{Indaziflam}

$\mathrm{O}$ indaziflam $(\mathrm{N}-[(1 \mathrm{R}, 2 \mathrm{~S})-2,3$-dihidro2,6-dimetil-1H-inden-1-il]-6-[(1R)-1-

fluoroetilo]-1,3,5-triazina-2,4-diamina) é um novo herbicida que oferece possibilidade de uso principalmente em pré-emergência de plantas daninhas Liliopsidas e Magnoliopsidas. Seu primeiro registro foi obtido em 2010 nos Estados Unidos para uso em gramados (Kaapro \& Hall, 2012). No Canadá, apresenta registro para o controle de plantas daninhas em áreas cultivadas com espécies frutíferas como maçãs, pera, pêssego, citros, uvas e nozes (Bayer, 2012). Possui amplo espectro de ação e duradoura eficácia mesmo com a aplicação de baixas doses. Para o controle de gramíneas anuais suscetíveis, as doses oscilam de 25 a $100 \mathrm{~g} \mathrm{ha}^{-1}$, podendo chegar a $150 \mathrm{~g} \mathrm{ha}^{-1} \mathrm{em}$ espécies mais tolerantes (Myers et al., 2009; Kaapro \& Hall, 2012). No Brasil, este herbicida encontra-se em fase de desenvolvimento para as culturas da cana-deaçúcar, citros e café.

$\mathrm{O}$ indaziflam pertence a também nova classe química denominada alkylazine. Atua na biossíntese de parede celular e é considerado o 
mais potente inibidor da parede celular já descoberto (Myers et al., 2009; Kaapro \& Hall, 2012). O mecanismo de ação exato deste herbicida ainda não está completamente esclarecido, no entanto, sabe-se que evita que novas células da parede celular sejam formadas, havendo então a paralisação do crescimento da planta. A formação de parede celular é inibida, mas a síntese de polímeros de polissacarídeos não é afetada. A inibição provavelmente ocorre em algum ponto na etapa da reticulação das microfibrilas de celulose. A inibição da divisão celular do tecido meristemático também tem sido proposta, como um modo de ação secundário (Griffin, 2005). Este herbicida também inibe a deposição de cristais na parede celular, afetando severamente a sua formação, a divisão e o alongamento das células. Isso indica que as folhas completamente desenvolvidas dificilmente serão afetadas pelo indaziflam, uma vez que a formação da parede celular já está concluída e nenhuma nova síntese de celulose ocorre. A eficácia em aplicações em pós-emergência tem sido observada até o estádio de duas folhas (Kaapro \& Hall, 2012). Brosnan et al. (2012) também sugerem que o tamanho da planta, o seu estádio de desenvolvimento e as condições ambientais podem limitar o controle do indaziflam em pós-emergência.

Estudos desenvolvidos por Brosnan \& Breeden (2012) demonstraram que a aplicação de indaziflam $\left(52,5 \mathrm{~g} \mathrm{ha}^{-1}\right)$ em pós-emergência inicial de Digitaria ischaeemum e Poa annua promoveu controle de $6 \%$ e $0 \%$, respectivamente, aos 35 dias após a aplicação (DAA). Quando foi aplicado em préemergência destas espécies o controle foi superior a 90\%, mesmo aos 195 DAA (Brosnan et al., 2011; Brosnan et al., 2012). Perry et al. (2011) também verificaram controle satisfatório $(\geq 80 \%)$ de $D$. ischaeemum e P. annua com a aplicação de 60 $\mathrm{g} \mathrm{ha}^{-1}$ de indaziflam em pré-emergência.
Trabalhos conduzidos no Brasil demonstraram que a aplicação de indaziflam em doses a partir de $100 \mathrm{~g} \mathrm{ha}^{-1}$ em préemergência das espécies Ageratum conyzoides, Sida rhombifolia, Digitaria horizontalis e Bidens pilosa promoveu controle satisfatório por período de até 120 dias após a aplicação (Christoffoleti et al., 2012; Nicolai et al., 2012a).

A seletividade deste herbicida também vem sendo estudada por pesquisadores brasileiros, principalmente em relação a efeitos de longo prazo que possam eventualmente ser observados em culturas perenes. As culturas do cafeeiro (Blanco \& Ramos, 2012a) e de citros (Blanco \& Ramos, 2012b; Nicolai et al., 2012b; Blanco et al., 2012) não apresentaram qualquer injúria após a aplicação de doses entre 75 e $150 \mathrm{~g} \mathrm{ha}^{-1}$.

No entanto, trabalhos conduzidos durante o desenvolvimento inicial das culturas do milho, milheto, sorgo, soja, girassol, algodão, beterraba e pepino indicaram que todas as espécies foram sensíveis à semeadura em solo que recebeu aplicação do indaziflam. O único sintoma observado nas diferentes espécies após o plantio em solo contendo este herbicida foi a não emergência das plântulas, exceto para o girassol. Algodão e milho não emergiram somente quando semeados no solo com a maior dose de indaziflam (100 $\left.\mathrm{g} \mathrm{ha}^{-1}\right)$. Para a soja observou-se a emergência no solo tratado com as duas menores doses (20 e $40 \mathrm{~g}$ $\mathrm{ha}^{-1}$ ), apesar das plantas morreram depois de alguns dias. Enquanto, para o sorgo, milheto, pepino e beterraba não houve emergência nem mesmo em solo que recebeu a menor dose deste herbicida (20 $\left.\mathrm{g} \mathrm{ha}^{-1}\right)$ (Guerra et al., 2014).

Jhala et al. (2012), realizando trabalhos de dose-resposta de indaziflam sobre Lolium multiflorum, verificaram que 3,65 e 14,62 $\mathrm{g} \mathrm{ha}^{-}$ ${ }^{1}$ são suficientes para inibir a germinação desta espécie em 70 e $90 \%$, respectivamente. Doses superiores a 29,2 $\mathrm{g} \mathrm{ha}^{-1}$ inibiram totalmente a germinação do L. multiflorum. 
As características físico-químicas do indaziflam também encontram-se na Tabela 1. Apesar das baixas doses de aplicação, este apresenta uma longa atividade residual para a maioria dos usos propostos, como resultado da sua longa persistência no solo (U.S. EPA, 2010). Segundo Jones et al. (2013), o aumento no teor de carbono orgânico do solo promove redução nas lesões foliares e radiculares de híbridos de grama-bermuda (Cynodon dactylon) expostos ao indaziflam. Este resultado corroboram com os descritos por Alonso et al. (2011), que verificaram correlação positiva entre a sorção do indaziflam e o conteúdo de carbono orgânico de diversos solos brasileiros. Embora o indaziflam seja um herbicida com características de ácido fraco (pKa 3,5) não se observou correlação entre a sorção e o pH do solo (Alonso et al., 2011). Esta falta de correlação pode ter ocorrido em função da estreita faixa de $\mathrm{pH}$ dos solos estudados $(\mathrm{pH}$ 5,4 - 6,5), na qual o indaziflam está substancialmente ionizado (> 99\%).

Jhala et al. (2012) conduziram ensaios de lixiviação do indaziflam, utilizando $L$. multiflorum como bioindicador, em solos com $4 \%$ de argila e $0,5 \%$ de matéria orgânica. Verificaram que quando se utilizou $73 \mathrm{~g} \mathrm{ha}^{-1} \mathrm{de}$ indaziflam, houve lixiviação até os 15, 20 e 25 $\mathrm{cm}$ de profundidade para as lâminas de precipitação de 50, 100 e 150 mm, respectivamente. Para a maior dose do herbicida (145 $\left.\mathrm{g} \mathrm{ha}^{-1}\right)$ a lixiviação ocorreu até 20,25 e $30 \mathrm{~cm}$. Estes resultados sugerem correlação positiva entre a dose de indaziflam e o volume de chuva com a lixiviação deste herbicida em solos de textura arenosa. Para estas condições, o indaziflam é considerado um herbicida com moderada potencialidade de lixiviação.

Estudos de Jhala \& Singh (2012) também demonstraram que o indaziflam apresentou lixiviação até $12,5 \mathrm{~cm}$ de profundidade após a simulação de $50 \mathrm{~mm}$ de precipitação em solo com $4 \%$ de argila e $0,5 \%$ de matéria orgânica, apresentando mobilidade semelhante aos herbicidas norflurazon e pedimentahlin. Quando a lâmina de irrigação foi de $150 \mathrm{~mm}$, este atingiu $27,2 \mathrm{~cm}$ de profundidade, sendo inferior à mobilidade observada para os herbicidas norflurazon e bromacil e superior a de simazine, diuron e pedimenthalin. Em solos do Brasil, o indaziflam pode ser considerado lixiviável apenas para o menor $\mathrm{K}_{\mathrm{oc}}\left(434 \mathrm{~L} \mathrm{~kg}^{-1}\right)$ quando a meia-vida for $>106$ dias, ao passo que para o maior valor de $\mathrm{K}_{\mathrm{oc}}\left(1544 \mathrm{~L} \mathrm{~kg}^{-1}\right)$ a meia vida teria de ser > 2036 dias (Alonso et al., 2011).

Os metabólitos deste herbicida são mais móveis do que a sua molécula original, e foram detectadas em estudos de campo em maiores profundidades $(105-120 \mathrm{~cm})$ (U.S. EPA, 2011). Portanto, os metabólitos do indaziflam têm maior potencial de lixiviação para a água subterrânea.

\section{Considerações Finais}

O aminocyclopyrachlor e indaziflam são novas moléculas que apresentam grande potencial para o controle de plantas daninhas em pré-emergência, pois proporcionam prolongado período de atividade residual.

$\mathrm{O}$ aminocyclopyrachlor é seletivo às espécies pertencente à família Poaceae, podendo ser uma alternativa interessante para o controle de plantas daninhas folhas largas nessas culturas. Já o indaziflam é muito pouco seletivo para culturas anuais, e seu uso tem sido dirigido principalmente a culturas semiperenes ou perenes.

Quanto ao comportamento no solo verifica-se correlação positiva com a sorção e o conteúdo de matéria orgânica. A longa persistência da atividade residual destas duas moléculas no solo exige que ambos sejam utilizados com cautela, pois podem trazer prejuízos para culturas em sucessão.

A maioria das informações a respeito destes herbicidas foi obtida em outros países, desta forma, é muito importante que antes da sua utilização em áreas comerciais sejam 
realizados estudos para verificar a seletividade, eficácia e comportamento destes herbicidas nas condições edafoclimáticas brasileiras.

\section{Referências}

AGBAKODA, C.S.O.; GOODIN, J.R. Picloram enhances 2,4-D movement in field bindweed. Weed Science, v.18, n.1, p.19-21, 1970.

ALONSO, D.G. et al. Sorption-desorption of indaziflam in selected agricultural soils. Journal of Agricultural and Food Chemistry, v.59, n.4, p.3096-3101, 2011.

ARMEL, G. R. et al. Comparisons of the experimental herbicide DPX-KJM44 with aminopyralid for control of key invasive weeds in Tennessee. Proceedings Weed Science Society of America Abstracts, v.49, n.410, 2009, p.410.

BAYER ENVIRONMENTAL SCIENCE, Indaziflam,15p. 2012. Disponível em: <http:// www.agf.gov.bc.ca/pesticides/indaziflam\%205 00_label.pdf $>$. Acesso em: 10 set. 2013.

BAYER ENVIRONMENTAL SCIENCE. Specticle herbicide technical bulletin; 2010, $16 \mathrm{p}$.

BELL, J.L.; BURKE, I.C.; PRATHER, T.S. Uptake, translocation and metabolism of aminocyclopyrachlor in prickly lettuce, rush skeletonweed and yellowstarthistle. Pest Management Science, v.67, n.5, p.1338-1348, 2011.

BLANCO, F.M.G. et al. Determinação da seletividade do herbicida indaziflam sobre mudas de citros avaliando dois tipos de transplantes. In: CONGRESSO BRASILEIRO DA CIÊNCIA DAS PLANTAS DANINHAS, 28, 2012, Campo Grande. Resumos... Campo Grande: Sociedade Brasileira da Ciência das Plantas Daninhas, 2012, p.70-75.

BLANCO, F.M.G.; RAMOS, Y.G. Avaliação da seletividade do herbicida indaziflam sobre as raízes e parte epígeas da cultura de café cv.
Catuaí. Resultado do primeiro ano. In: CONGRESSO BRASILEIRO DA CIÊNCIA DAS PLANTAS DANINHAS, 28, 2012, Campo Grande. Resumos... Campo Grande: Sociedade Brasileira da Ciência das Plantas Daninhas, 2012a, p.54-59.

BLANCO, F.M.G.; RAMOS, Y.G. Avaliação da seletividade do herbicida indaziflam sobre as raízes e parte epígeas da cultura do citros cv. Valência. Resultado do primeiro ano. In: CONGRESSO BRASILEIRO DA CIÊNCIA DAS PLANTAS DANINHAS, 28, 2012, Campo Grande. Resumos... Campo Grande: Sociedade Brasileira da Ciência das Plantas Daninhas, 2012b, p.54-59.

BROSNAN, J.T. et al. Pre and post emergence annual bluegrass control with indaziflam. Weed Technology, v.26, n.1, p.48-53, 2012.

BROSNAN, J.T.; BREEDEN, G.K. Application placement affects postemergence smooth crabgrass (Digitaria ischaemum) and annual bluegrass (Poa annua) control with indaziflam Weed Technology, v.26, n.4, p.661-665, 2012.

BROSNAN, J.T.; Mc CULlUOGH, P.E.; BREEDEN, G.K. Smooth crabgrass control with indaziflam at various spring timings. Weed Technology, v.25, n.3, p.363-366, 2011.

BUKUN, B. et al. Absorption and translocation of aminocyclopyrachlor and aminocyclopyrachlor- methyl ester in Canada thistle (Cirsium arvense). Weed Science, v.58, n.2, p.96-102, 2010.

CHRISTOFFOLETI, P.J. et al. Indaziflam: Novo mecanismo de ação para a cana-deaçúcar. In: CONGRESSO BRASILEIRO DA CIÊNCIA DAS PLANTAS DANINHAS, 28, 2012, Campo Grande. Resumos... Campo Grande: Sociedade Brasileira da Ciência das Plantas Daninhas, 2012, p.76-80.

CLAUS, J.S. et al. Aminocyclopyrachlor development and registration uptade. North 
Central Weed Science Society Proceedings, v.64, n.128, 2009. p.303.

CLAUS, J.S.; TURNER, R.G.; HOLLIDAY, M. DuPont aminocyclopyrachlor (proposed common name) (DPX-MAT28/KJM44) herbicide for use in turf, IWC, bare-ground, and brush markets. International Weed Science Congress. v.5, n.277, 2008. p.645.

CONKLIN ,K.L.; LYM, R.G. Effect of temperature and moisture on aminocyclopyrachlor soil half-life. Weed Technology, v.27, n.3, p.552-556, 2013.

EDWARDS, R. The effects of DPX-KJM44 on native and non-native Colorado rangeland species. Proceeding Western Society Weed Science Abstracts, v.61, n.5, 2008.

FINKELSTEIN, B.L. et al. Discovery of aminocyclopyrachlor (proposed common name) (DPX-MAT28): a new broad spectrum auxinic herbicide. In: Proceedings of the 236th ACS National Meeting in Philadelphia, PA. Washington. Abstracts... American Chemical Society, 2008. p.19.

FLESSNER, M.L. et al. Anatomical response of St. Augustinegrass to aminocyclopyrachlor tratament. Weed Science, n.2, v.59, p.263-269, 2011.

FLINT, J L.; BARRETT, M. Effects of glyphosate combinations with 2,4-D or dicamba on field bindweed (Convolvulus arvensis). Weed Science, v.37, n.1, p.12-18, 1989.

GRIFFIN, J.L. Inhibition of cell wall synthesis. In: Weed Course. 2005, p.150-153.

GROSSMANN, K. Auxin herbicides: current status of mechanism and mode of action. Pest Management Science, v.66, n.2, p.113-120, 2009.

GUERRA, N. et al. Sensibility of plants species to herbicides aminocyclopyrachlor and indaziflam. Planta Daninha, 2014. No prelo.
JENKS, B.M. Yellow toadflax control in rangeland with DPX-MAT28. Proceeding Western Society Weed Science Abstracts, v.63, n.4, 2010. p.76.

JHALA, A.J.; RAMIREZ, A.H.; SINGH, M. Leaching of indaziflam applied of two rates under different rainfall situations in Florida Clandler Soil. Bulletin of Environmental Contamination and Toxicology, v.88, n.3, p.326-332, 2012.

JHALA, A.J.; SINGH, M. Leaching of indaziflam compared with residual herbicides commonly used by Florida citrus. Weed Technology, v.26, n.3, p.602-607, 2012.

JONES, P.A. et al. Effect of reed-sedge peat moss on hybrid bermudagrass injury with indaziflam and prodiamine in sand based root zones. Weed Technology, v.27, n.3, p.547$551,2013$.

KAAPRO, J.; HALL, J. Indaziflam, a new herbicide for pre-emergent control of weeds in turf, forestry, industrial vegetation and ornamentals. Pakistan Journal Weed Science Research, v.18, n.esp., p.267-270, 2012.

KNISS, A.R.; LYON, D.J. Winter wheat response to preplant applications of aminocyclopyrachlor. Weed Technology, v.25, n.1, p.51 -57, 2011.

LEWIS, D.F. et al. Absorption, translocation and metabolism of aminocyclopyrachlor in tall fescue (Lolium arundinaceum). Weed Science, v. 61, n.3, p.348-352, 2013.

LINDENMAYER, R.B. et al. Aminocyclopyrachlor absorption, translocation and metabolism in field bindweed (Convolvulus arvensis). Weed Science, v.61, n.1, p.63-67, 2013.

LINDENMAYER, R.B. Understanding aminocyclopyrachlor behavior in soil and plant. 2012. 82f. Tese de doutorado (Colorado State University). Colorado, 2012. 
LINDENMAYER, R.B.; WESTRA, P.P.; BRUNK, G. Selected invasive species control using aminocyclopyrachlor. Proceeding Western Society Weed Science Abstracts, v.62, n.42, 2009. p.42.

Mc CLOSKEY, W.B. et al. Knapweed management in southwestern abandoned pastures. Proceeding Weed Science Society of America Abstracts, v.63, n.43, 2011, p.58.

MYERS, D.F. et al. Indaziflam/BCS AA170717 - a new herbicide for preemergent control of grasses and broadleaves in turf and ornamentals. Proceeding South Society Weed Science Abstracts, v.62, p.393, 2009.

NICOLAI, M. et al. Avaliação da seletividade do herbicida indaziflam ao longo de três anos para a cultura do citros. In: CONGRESSO BRASILEIRO DA CIÊNCIA DAS PLANTAS DANINHAS， 28， 2012, Campo Grande. Resumos... Campo Grande: Sociedade Brasileira da Ciência das Plantas Daninhas, 2012b, p.81-85.

NICOLAI, $M$. et al. Indaziflam: Novo mecanismo de ação para o citros. In: CONGRESSO BRASILEIRO DA CIÊNCIA DAS PLANTAS DANINHAS, 28, 2012, Campo Grande. Resumos... Campo Grande: Sociedade Brasileira da Ciência das Plantas Daninhas, 2012a, p.76-80.

OLIVEIRA JR., R.S. et al. Comparative sorption, desorption and leaching potential of aminocyclopyrachlor and picloram. Journal of Environmental Science and Health, Part B v.48, p.1049-1057, 2012.

OLIVEIRA JR., R.S.; ALONSO, D.G.; KOSKINEN, W.C. Sorption-desorption of aminocyclopyrachlor in selected Brazilian soils. Journal of Agricultural and Food Chemistry, v.59, n.8, p.4045-4050, 2011.

PERRY, D.H. et al. Indaziflam utilization for controlling problematic turfgrass weeds. Plant Management Network, 2011. Disponível em: <http://www.plantmanagementnetwork.org/ pub/ats/research/ 2011/indaziflam/> Acesso em: 03 jun. 2013.

RICK, S.K.; TURNER, R.G.; MEREDITH, J.H. Biology review of aminocyclopyrachlor. Proceeding North Central Weed Science Society, v.63, p.202, 2008.

STRACHAN, S.D. et al. Correlation of chemical analysis of residual levels of aminocyclopyrachlor in soil to biological responses of alfalfa, cotton, soybean, and sunflower. Weed Technology, v.25, n.2, p.239-244, 2011.

STRACHAN, S.D. et al. Vapor movement of synthetic auxin herbicide: Aminocyclopyrachlor,

aminocyclopyrachlormethyl ester, dicamba and aminopyralid. Weed Science, v.58, n.2, p.103108, 2010.

STRACHAN, S.D.; FERRY, N.M.; COOPER, T.L. Vapor movement of aminocyclopyrachlor, aminopyralid and dicamba in the field. Weed Technology, v.27, n.1, p.143-155, 2013.

TURNER, R.G. et al. Aminocyclopyrachlor blend products for vegetation management on railroad and utility sites. Proceeding Society Range Management Proceeding Meeting v.64, n.130, 2010. p.130.

TURNER, R.G. et al. Technical introduction of the new DuPont vegetation management herbicide aminocyclopyrachlor. Proceeding South Society Weed Science Abstracts, v.62, p.405, 2009.

U.S. EPA. Pesticide fact sheet for indaziflam. 2011. Disponível em: http://www.epa.gov/ opprd001/factsheets/indaziflam.pdf. Acesso em: 04 jun. 2013.

U.S. EPA. Pesticide fact sheet, 2010. Conditional registration, 2010, 108p.

VASSIOS, J.D. et al. Native grass tolerance to aminopyralid and DPX-KJM44. Proceeding Weed Science Society of America Abstracts, v. 49, n.153, 2009. p.153. 
WESTRA, P. et al. Aminocyclopyrachlor for invasive weed management and restoration grass safety in the central great plains. Proceeding North Central Weed Science Society, v.63, p. 203, 2008.

WESTRA, P.; WILSON, R.; EDWARDS, M. Agronomic crop responses to KJM-44 herbicide. Proceeding North Central Weed

Science Society v.63, p.61-62, 2008. 